\title{
Glucose Urine Excretion Rate
}

National Cancer Institute

\section{Source}

National Cancer Institute. Glucose Urine Excretion Rate. NCI Thesaurus. Code 1117834.

A determination of the amount of glucose being excreted in urine over a defined period of time. 